Classification

Physics Abstracts

$75.50 \mathrm{~K}$

\title{
Sample to sample fluctuations in the random energy model
}

\author{
B. Derrida
}

Service de Physique Théorique, CEN Saclay, 91191 Gif-sur-Yvette Cedex, France

and G. Toulouse

E.S.P.C.I., 10, rue Vauquelin, 75231 Paris Cedex, France

(Reçu le 10 janvier 1985, accepté le 28 janvier 1985)

Résumé. - Dans la phase verre de spin, la théorie de champ moyen dit que les poids des vallées varient d'un échantillon à l'autre. On dérive des expressions exactes pour les lois de probabilité de ces fluctuations à partir du modèle à énergies aléatoires, sans recours à la méthode des répliques.

\begin{abstract}
In the spin glass phase, mean field theory says that the weights of the valleys vary from sample to sample. Exact expressions for the probability laws of these fluctuations are derived, from the random energy model, without recourse to the replica method.
\end{abstract}

The random energy model (RE) was introduced [1, 2] in 1980 as an extreme simplification of spin glass problems. Many properties, which were obtained exactly, appear qualitatively similar to those expected for the Sherrington-Kirkpatrick (SK) model [3].

A step further was made in 1984 by Gross and Mézard [4] showing that the replica method could be applied to the RE model. This had two important consequences. For the first time, some results of the replica trick could be checked against an alternate derivation, in the context of replica symmetry breaking. Furthermore, it became clear that some subtle features derived for the SK model, from the Parisi Ansatz, were essentially present in the RE model.

For those who never felt that the replica trick should be taken as a final word, the RE model offered then the best hope for matching and even outpassing the procedures of the replica method, as applied to spin glasses. This paper fulfils these hopes, in deriving an analytical formulation for the lack of self averaging, the fluctuations from sample to sample of the spin glass order parameter, which are one of the most conspicuous predictions of the theory $[5,6]$ and one which is accessible to numeral checks [7, 8].

Using the Parisi Ansatz [5, 6] for the SK model, one can study the following quantity

$$
Y=\sum_{s} W_{s}^{2}
$$

where $W_{s}$ is the weight of the sth free energy valley and the sum(1) runs over all the valleys. 
It was recently discovered $[5,6]$ that $Y$ is not a self averaging quantity, i.e. that in the thermodynamic limit, $Y$ is sample dependent. Therefore the quantity which is meaningful to consider is the probability distribution $\Pi(Y)$ of $Y$. It was found that $\Pi(Y)$ is a function of only its average $y$.

$$
\int_{0}^{1} \Pi(Y) Y \mathrm{~d} Y=y
$$

The first moments of $\Pi(Y)$ were calculated and they are polynomials in $y$. Therefore it is a universal object since it depends on all the parameters (temperature, magnetic field...) through $y$ only. The purpose of the present paper is to calculate $\Pi(Y)$ directly, without using the replica method. We take advantage of the universality of $\Pi(Y)$ to capture it on the easiest ground, namely the RE model.

The RE model $[1,2]$ can be defined in the following way : it is a system of $2^{N}$ energy levels $E_{\alpha}$ which are independent random variables distributed according to a probability distribution $\rho\left(E_{\alpha}\right)$ :

$$
\rho\left(E_{\alpha}\right)=\left(N \pi J^{2}\right)^{-\frac{1}{2}} \cdot \exp \left(-\frac{E_{\alpha}^{2}}{N J^{2}}\right) .
$$

In the thermodynamic limit, the random energy model undergoes a phase transition at a temperature $T_{\mathrm{c}}$

$$
T_{\mathrm{c}}=\frac{J}{2 \sqrt{\log 2}}
$$

and the free energy in the low temperature phase is

$$
-\frac{F}{T}=\lim _{N \rightarrow \infty} \frac{\langle\log Z\rangle}{N}=\frac{J}{T} \sqrt{\log 2} .
$$

Although in the thermodynamic limit, the free energy per spin reduces to the ground state energy per spin, one can try to study the statistical weights of the energy levels which are close to the ground state.

Since the energy levels are independent random variables, each energy level forms its own free energy valley. Therefore, the weight $W_{\alpha}$ of the level $\mathrm{E}_{\alpha}$ is just

$$
W_{\alpha}=\frac{\mathrm{e}^{-\beta E_{\alpha}}}{\sum_{\gamma} \mathrm{e}^{-\beta E_{\gamma}}}
$$

and $Y$ is given by

$$
Y=\sum_{\alpha} W_{\alpha}^{2}=\frac{\sum_{\alpha} \mathrm{e}^{-2 \beta E_{\alpha}}}{\left(\sum_{\gamma} \mathrm{e}^{-\beta E_{\gamma}}\right)^{2}}=\frac{Z(2 \beta)}{[Z(\beta)]^{2}} .
$$

We shall now calculate all the moments of $Y$. Our final result will be that

$$
\left\langle Y^{v}\right\rangle=\frac{(-1)^{n+1} \cdot T_{\mathrm{c}} / T}{\Gamma(2 v) \cdot \Gamma(n-v)} \cdot \int_{0}^{\infty} \mu^{n-1-v} \cdot \frac{\mathrm{d}^{n} \log g}{\mathrm{~d} \mu^{n}} \cdot \mathrm{d} \mu
$$


where $n$ is an integer defined by

$$
n-1<v<n
$$

and the function $g(\mu)$ is

$$
g(\mu)=\int_{0}^{\infty}\left(1-\mathrm{e}^{-u-\mu u^{2}}\right) \cdot u^{-\frac{T}{T_{c}}-1} \cdot \mathrm{d} u .
$$

For positive integer moments the integral over $\mu$ is dominated by $\mu=0$ and formula (8) becomes

$$
\left\langle Y^{n}\right\rangle=\left.\frac{(-1)^{n+1} T_{\mathrm{c}} / T}{\Gamma(2 n)} \cdot \frac{\mathrm{d}^{n} \log g}{\mathrm{~d} \mu^{n}}\right|_{\mu=0} .
$$

Let us now derive equation (8). The method we shall follow is similar to the one which was used in the Appendix B of reference [2]. For any positive $v$, one can write

$$
\begin{aligned}
& Z^{v}(2 \beta)=\frac{1}{\Gamma(n-v)} \cdot \int_{0}^{\infty}[Z(2 \beta)]^{n} \cdot \mathrm{e}^{-t_{1} Z(2 \beta)} \cdot t_{1}^{n-v-1} \cdot \mathrm{d} t_{1} \\
& Z^{-2 v}(\beta)=\frac{1}{\Gamma(2 v)} \cdot \int_{0}^{\infty} \mathrm{e}^{-t Z(\beta)} \cdot t^{2 v-1} \cdot \mathrm{d} t
\end{aligned}
$$

where $n$ is defined by (9).

If we make the change of variable $t_{1}=t^{2} \mu$, one can show that

$$
\left\langle\left[\frac{Z(2 \beta)}{(Z(\beta))^{2}}\right]^{v}\right\rangle=\frac{(-1)^{n}}{\Gamma(2 v) \cdot \Gamma(n-v)} \cdot \int_{0}^{\infty} \frac{\mathrm{d} t}{t} \int_{0}^{\infty} \mu^{n-1-v} \cdot \mathrm{d} \mu \cdot \frac{\mathrm{d}^{n}}{\mathrm{~d} \mu^{n}}\left\langle\mathrm{e}^{-t Z(\beta)-\mu t^{2} Z(2 \beta)}\right\rangle .
$$

The main advantage of representation (14) is that the average in the right hand side of (14) can be done easily because the quantity to average is a product of $2^{N}$ independent random variables.

If we define $\phi(t, \mu)$ by

$$
\exp (-\phi)=\left\langle\exp \left(-t Z(\beta)-\mu t^{2} Z(2 \beta)\right)\right\rangle
$$

then one has

$$
\exp \left(-\frac{\phi}{2^{N}}\right)=\int_{-\infty}^{+\infty} \frac{\mathrm{d} E}{\sqrt{N \pi J^{2}}} \cdot \exp \left(-\frac{E^{2}}{N J^{2}}-t \mathrm{e}^{-\beta E}-\mu t^{2} \mathrm{e}^{-2 \beta E}\right) .
$$

If we define $\lambda$ by

$$
\lambda=\sqrt{N} \beta J
$$

one can show by a method similar to the one used in reference [2] that in the limit $\lambda \rightarrow \infty$

$$
\frac{\phi}{2^{N}} \simeq \frac{1}{\sqrt{\pi}} \cdot \frac{1}{\lambda} \cdot \exp \left(-\frac{\log ^{2} t}{\lambda^{2}}\right) \cdot \int_{0}^{\infty}\left(1-\mathrm{e}^{-u-\mu u^{2}}\right) \cdot u^{\frac{2 \log t}{\lambda^{2}}-1} \cdot \mathrm{d} u
$$


provided that the ratio $\log t / \lambda^{2}$ remains finite and fulfils the following condition

$$
-\frac{1}{2}<\frac{\log t}{\lambda^{2}}<0 \text {. }
$$

To avoid a long discussion we shall not consider other ranges of values of $\log t$. For $0<T<T_{\mathrm{c}}$, only the region defined by (19) gives a finite contribution to the integral (14).

Using the fact that $\lambda$ is large, one can calculate $\log t$ from (18)

$$
\log t=-\frac{N J}{T} \sqrt{\log 2}+\frac{T_{\mathrm{c}}}{T}\left[\frac{1}{2} \log N+\log \phi-\log (g(\mu))+\frac{1}{2} \log \pi-\log \frac{T}{J}\right]+\mathcal{O}(1)
$$

where $g(\mu)$ is defined by

$$
g(\mu)=\int_{0}^{\infty}\left(1-\mathrm{e}^{-u-\mu u^{2}}\right) \cdot u^{-\frac{T}{T_{c}}-1} \cdot \mathrm{d} u
$$

The condition (19) becomes then simply

$$
0<\frac{T}{T_{\mathrm{c}}}<1
$$

One sees that (20) expresses that

$$
\phi=A \cdot t^{T / T_{\bullet}} \cdot g(\mu)
$$

where $A$ is a constant which can be easily obtained from (20).

We can now come back to (14). Using (15) and (23), one sees that

$$
\left\langle\left[\frac{Z(2 \beta)}{(Z(\beta))^{2}}\right]\right\rangle=\frac{(-1)^{n}}{\Gamma(2 v) \Gamma(n-v)} \cdot \int_{0}^{\infty} \frac{\mathrm{d} t}{t} \int_{0}^{\infty} \mu^{n-1-v} \cdot \mathrm{d} \mu \cdot \frac{\mathrm{d}^{n}}{\mathrm{~d} \mu^{n}} \exp \left[-A \cdot t^{T / T_{\mathrm{c}}} \cdot g(\mu)\right] .
$$

The integral over $t$ can be done easily if one realizes that

$$
\begin{aligned}
\int_{0}^{\infty} \frac{\mathrm{d} t}{t} \cdot \frac{\mathrm{d}^{n}}{\mathrm{~d} \mu^{n}} \exp \left[-A \cdot t^{T / T_{\mathrm{c}}} \cdot g(\mu)\right] & =\int_{0}^{\infty} \frac{\mathrm{d} t}{t} \cdot \frac{\mathrm{d}^{n}}{\mathrm{~d} \mu^{n}}\left[\exp \left(-A \cdot t^{T / T_{\mathrm{c}}} \cdot g(\mu)\right)-\exp \left(-A \cdot t^{T / T_{\mathrm{c}}}\right)\right] \\
& =-\frac{T_{\mathrm{c}}}{T} \cdot \frac{\mathrm{d}^{n}}{\mathrm{~d} \mu^{n}} \log [g(\mu)]
\end{aligned}
$$

Therefore one gets the announced expression (8).

Since the support of $\Pi(Y)$ is bounded, there is a unique measure $\Pi(Y)$ whose moments are given by (8). One can calculate several generating functions of $\Pi(Y)$.

A simple one is

$$
\int_{0}^{1} \mathrm{~d} Y \cdot \Pi(Y) \cdot \psi(4 \mu Y)=1-2 \frac{T_{\mathrm{c}}}{T} \mu \frac{\mathrm{d} \log g}{\mathrm{~d} \mu}
$$


where

$$
\psi(x)=\frac{2}{\sqrt{x}} \cdot \mathrm{e}^{\frac{1}{x}} \cdot \int_{\frac{1}{\sqrt{x}}}^{\infty} \mathrm{e}^{-t^{2}} \cdot \mathrm{d} t .
$$

The expansion of (26) around $\mu=0$ gives the moments $\left\langle Y^{n}\right\rangle$ for $n$ positive whereas the expansion for $\mu$ large gives the moments $\left\langle Y^{-n / 2}\right\rangle$. Since all the moments depend only on the ratio $T / T_{\mathrm{c}}$, one sees that $\Pi(Y)$ is only a function of $y$

$$
y=\langle Y\rangle=1-\frac{T}{T_{\mathrm{c}}} .
$$

For the first moments, one recovers the results given in reference [6]. One can also calculate easily the moments $\left\langle Y^{-n / 2}\right\rangle$. For example one finds for $\left\langle Y^{-1 / 2}\right\rangle$

$$
\left\langle Y^{-\frac{1}{2}}\right\rangle=\frac{1}{\sqrt{\pi}} \cdot \frac{\Gamma\left(\frac{y}{2}\right)}{\Gamma\left(\frac{1+y}{2}\right)} .
$$

All the results given above can easily be generalized. If we define

$$
Y=\sum_{\alpha}\left(W_{\alpha}\right)^{\delta}=\frac{Z(\delta \beta)}{[Z(\beta)]^{\delta}}
$$

for any $\delta$, one can repeat the calculation which was done above in the special case $\delta=2$.

The result is

$$
\left\langle\left[\frac{Z(\delta \beta)}{(Z(\beta))^{\delta}}\right]^{v}\right\rangle=\frac{(-1)^{n+1} T_{\mathrm{c}} / T}{\Gamma(\delta v) \cdot \Gamma(n-v)} \cdot \int_{0}^{\infty} \mu^{n-1-v} \cdot \mathrm{d} \mu \cdot \frac{\mathrm{d}^{n}}{\mathrm{~d} \mu^{n}} \log \left[G_{\delta}(\mu)\right]
$$

where $n$ is defined by $(9)$ and $G_{\delta}(\mu)$ is given by

$$
G_{\delta}(\mu)=\int_{0}^{\infty}\left(1-\mathrm{e}^{-u-\mu u^{\delta}}\right) \cdot u^{-\frac{T}{T_{c}}-1} \cdot \mathrm{d} u .
$$

The generalization of (26) is

$$
\int_{0}^{1} \mathrm{~d} Y \cdot \Pi_{\delta}(Y) \cdot Q_{\delta}(\mu Y)=1-\delta \cdot \frac{T_{\mathrm{c}}}{T} \cdot \frac{\mathrm{d} \log G_{\delta}}{\mathrm{d} \log \mu}
$$

where $Q_{\delta}(x)=\int_{0}^{\infty} \mathrm{d} s \cdot \mathrm{e}^{-s-x s^{\delta}}$. For $\delta=2$, the previous results are recovered, with $\Pi_{2}(Y)=\Pi(Y)$. From the moments of $\Pi_{\delta}(Y)$, the correlated probability of finding two states of weights $W_{1}$ and $W_{2}$ in the same sample is extracted as

$$
\begin{array}{r}
P\left(W_{1}, W_{2}\right)=W_{1} P\left(W_{1}\right) \delta\left(W_{1}-W_{2}\right)+(1-y) \cdot \frac{\left(W_{1} W_{2}\right)^{-1+y}\left(1-W_{1}-W_{2}\right)^{1-2 y}}{\Gamma^{2}(y) \Gamma(2-2 y)} \times \\
\times \theta\left(1-W_{1}-W_{2}\right) .
\end{array}
$$


where $P(W)=\frac{W^{-1+y}(1-W)^{-y}}{\Gamma(y) \Gamma(1-y)}$ is the probability of finding a state of weight $W$. Generalization to higher order correlations is straightforward. All this [6] follows from energy randomness.

With these exact results, a strategy for entering the post-replica age in spin glass physics becomes clear. Firstly, derive a physically complete knowledge of the random energy model, analytical along the preceding lines. Secondly, show how, via some renormalization procedure, this model can be seen as a sort of fixed point for the mean field theory of spin glasses.

\section{Acknowledgments.}

We thank N. Sourlas and J. Vannimenus for helpful conversations. Our friends in Rome, M. Mézard, G. Parisi, M. Virasoro have been making way toward the same goal and we have learnt recently that they have reached similar conclusions. In agreement with them, we hope that our independent treatments will be found complementary and helpful to the reader.

\section{References}

[1] Derrida, B., Phys. Rev. Lett. 45 (1980) 79.

[2] Derrida, B., Phys. Rev. B 24 (1981) 2613.

[3] Sherrington, D. and KirkPatrick, S., Phys. Rev. Lett. 35 (1975) 1972 ; Phys. Rev. B 17 (1978) 4384.

[4] Gross, D. and Mézard, M., Nucl. Phys. B 240 (FS12) (1984) 431.

[5] Mézard, M., Parisi, G., Sourlas, N., Toulouse, G. and Virasoro, M., Phys. Rev. Lett. 52 (1984) 1146.

[6] Mézard, M., Parisi, G., Sourlas, N., Toulouse, G. and Virasoro, M., J. Physique 45 (1984) 843.

[7] Parga, N., Parisi, G. and Virasoro, M., J. Physique Lett. 45 (1984) L-1063.

[8] Young, A. P., Bray, A. J., Moore, M. A., J. Phys. C 17 (1984) L149. 